\title{
AD-0974 - A 2-3 years follow-up study on fertility after laparoscopy among infertile diabetic women in a tertiary care hospital
}

\author{
Dr. Naushaba Tarannum Mahtab, Dr. Nusrat Mahmud, Dr. Tanzeem Sabina Chowdhury, \\ Prof. T. A. Chowdhury \\ Department of Obstetrics and Gynaecology, BIRDEM General Hospital-2
}

\begin{abstract}
Background:
Once a couple passes twelve months of trying to get pregnant with no success, and without the use of contraception, the couple is deemed infertile. When the couple seeks medical help, one of the first conditions the doctor will look for is diabetes, since the condition can cause fertility problems.
\end{abstract}

According to the American Diabetes Association, there are more than 200,000 new cases of Type II diabetes diagnosed every year, with another $2.4 \%$ of the general childbearing population suffering from the disease but not knowing it.

\begin{abstract}
Aims:
With the rates of Type II diabetes rising every year, more and more infertility specialists are looking toward this health issue as a main cause of some otherwise unexplained infertility cases they see. Type 2 diabetes is associated with infertility and alterations in the length of menstrual cycle. This may be explained by the correlation of Type 2 diabetes to polycystic ovarian syndrome (PCOS), which is highly prevalent in women with Type 2 diabetes.
\end{abstract}

Routine use of diagnostic laparoscopy for the evaluation of all cases of female infertility is currently under debate. Until now, laparoscopy is a widely used diagnostic and therapeutic means of treating infertility.

This study investigates the fertility outcome for women up to 2 to 3 years after laparoscopic management. The null hypothesis of this study is that infertility and pregnancy outcomes in women with diabetes after laparoscopy are identical to those of non-diabetic control subjects.

\section{Method:}

From May 2013 to November 2014, a total of 303 infertile women undergoing laparoscopy at BIRDEM hospital, were asked to participate in the study. 60 subjects were lost to follow up during 2016. Among the 243 study subjects, 45 (18.5\%) were known to have diabetes mellitus.

\section{Results:}

Average age at the time of laparoscopy was $29.86 \pm 4.6$ years (range 21 - 43) in both the study groups. The mean BMI (Body Mass Index) among the diabetic group was $27.8 \pm 6.09$, which was almost similar to the non-diabetic group $(26.21 \pm 5.01)$. After laparoscopy, it was observed that among the 243 follow up patients, 51 patients had diagnostic laparoscopy and 136 underwent laparoscopic ovarian drilling (LOD). The patients who underwent LOD, 25 (18.4\%) of them were diabetic; there was no significant difference between the study groups. From the time of index surgery to follow-up, 40.3\% (98/243) of women who had tried to conceive had home pregnancy test positive and $25.5 \%(62 / 243)$ had a live birth following their surgery. Among these 62 subjects with live birth, only 8 (12.9\%) of them were diabetic. In the diabetic study group after 2 to 3 years of laparoscopy, 30 (20.7\%) remained nulliparous and 5 (17.9\%) had abortion. However, none of the tests were statistically significant.

\section{Discussion:}

In our day to day clinical practice, it is not always possible to justify when to offer laparoscopy in fertility work-up. According to data published in retrospective non-controlled studies, diagnostic laparoscopy after several failed cycles of ovulation induction enables the detection of a significant proportion of pelvic pathology amenable to treatment. In a recent publication from RCOG green top guideline, laparoscopic ovarian drilling was labeled as second line of treatment in PCOS.

In general, the presence of infertility needs much attention regarding identification and treatment of the problem. In the diabetic population infertility may add to the social strain of daily diabetic care and to potential diabetic complications. In these women early discovery by laparoscopy of an infertility problem is especially important. Also it has been shown that laparoscopic treatment can give excellent results regarding infertility as well as menstrual disturbances.

The current study showed follow-up of 243 infertile women who underwent laparoscopy two to three years prior to the follow-up. The results after laparoscopy showed that, the diabetic women and controls have the same cumulative rate of pregnancy, which suggests that the time to conceive is similar in the two groups. There was no trend towards a lower frequency of pregnancies in infertile diabetic women compared with infertile controls.

It can be concluded that after laparoscopy, fertility in diabetic women seems to be similar to that of the general population. However, with adequate sample size of diabetic and non-diabetic women, a future study may reveal significant findings.



\section{References}

Kjaer K, Hagen C, Sandø SH, Eshøj O. Infertility and pregnancy outcome in an unselected group of women with insulin-dependent diabetes mellitus. American journal of obstetrics and gynecology. 1992 May 1;166(5):1412-8.

Livshits A, Seidman DS. Fertility issues in women with diabetes. Women's Health. 2009 Nov;5(6):701-7. 